\title{
Efeitos do ciclismo no sistema musculoesquelético em um grupo amador em
}

\section{Santarém-PA}

\author{
Effects of cycling on the musculuskeletal system in an amateur group in Santarém-PA \\ Efectos del ciclismo en el sistema musculuesquelético en grupo de aficionados en Santarém-PA
}

Recebido: 10/11/2021 | Revisado: 15/11/2021 | Aceito: 15/11/2021 | Publicado: 27/11/2021

\author{
Zelinda Nogueira Carvalho \\ ORCID: https://orcid.org/0000-0002-4230-9706 \\ Centro Universitário da Amazônia, Brasil \\ E-mail: zlindacarvalho@gmail.com \\ Caroline dias de Sousa \\ ORCID: https://orcid.org/0000-0001-9960-5074 \\ Centro Universitário da Amazônia, Brasil \\ E-mail: caroline.dias.sousa92gmail.com \\ Assis Júnior Cardoso Pantoja \\ ORCID: https://orcid.org/0000-0002-8301-3135 \\ Centro universitário da Amazônia, Brasil \\ E-mail: assisfisio_jr@hotmail.com
}

\begin{abstract}
Resumo
A bicicleta é usada como meio de transporte e laser. É de fácil acesso e uma alternativa de locomoção favorável, além de ter um custo-benefício aceitável e promover uma boa saúde para seus praticantes. Como todo esporte, de alguma forma, há um algum fator que leva a riscos de lesões. Este estudo buscou analisar os efeitos musculoesqueléticos que ocorrem em ciclistas amadores em um grupo na cidade de Santarém/PA. A pesquisa ocorreu em Santarém no estado do Pará com um grupo organizado de ciclistas amadores, que pedalam de forma regular semanalmente e o estudo foi composto por 18 ciclistas. Como ferramenta para coleta dos dados, foram aplicados on-line um questionário já existente sobre qualidade de vida pelo SF-36 que é um instrumento traduzido por Cicinelli et al. (1999) e o questionário Nórdico Músculo/esquelético de Kourinka et al. (1989) Os dados obtidos foram analisados através da estatística descritiva quantitativa, com uso de tabelas de frequência. Na amostra, o gênero feminino teve maior participação do que o gênero masculino, uma vez que $56 \%$ eram mulheres. Conclui-se que o perfil dos ciclistas, membros da amostra, evidencia que regiões anatômicas da coluna lombar e as regiões dos joelhos foram as áreas que mais predominaram para queixa de dores ao se comparar com as demais regiões. Além disso, constatou-se que a região torácica é uma área pouco relevante para lesões nesses ciclistas amadores e a amostra demonstrou-se não ter nenhuma dificuldade ou limitação em praticar suas atividades rigorosas do dia a dia ou modalidades esportivas.
\end{abstract}

Palavras-chave: Ciclismo; Atletas amadores; Lesões musculoesqueléticas.

\begin{abstract}
The bicycle is used as a means of transport and laser is easily accessible and also a favorable alternative for transportation, in addition to having an acceptable cost-benefit ratio and for promoting good health for its riders. As with every sport, there is somehow a factor that leads to an injury risk. This study aimed to analyze the musculoskeletal effects that occur in amateur cyclists in a group in the city of Santarém/PA. The research took place in Santarém, Pará, with an organized group of amateur cyclists who cycle regularly on a weekly basis and the study consisted of 18 cyclists. As a tool for data collection, an existing questionnaire on quality of life using the SF-36, which is a global instrument created by Ciconelli et al. (1999) and the Nordic Muscle/Skeletal questionnaire by Kourinka et al. (1989). The data obtained were analyzed using quantitative descriptive statistics, using frequency tables. In the sample, females had greater participation than males, since $56 \%$ were women. It is concluded that the profile of cyclists who are members of the sample shows that anatomical regions of the lumbar spine and knee regions were the most predominant areas for complaints of pain when compared to the other regions and that the thoracic region proved to be a poor area. relevant to injuries in these amateur cyclists and that the sample showed no difficulty or limitation in practicing their rigorous activities of the day or sports.
\end{abstract}

Keywords: Cycling; Amateur athletes; Musculoskeletal injuries.

\section{Resumen}

La bicicleta se utiliza como medio de transporte y el láser es de fácil acceso y también una alternativa favorable para el transporte, además de tener una relación costo-beneficio aceptable y promover la buena salud de sus ciclistas. Como ocurre con todos los deportes, existe de alguna manera un factor que conduce al riesgo de lesiones. Este 
estudio tuvo como objetivo analizar los efectos musculoesqueléticos que se producen en ciclistas aficionados en un grupo de la ciudad de Santarém / PA. La investigación se llevó a cabo en Santarém, Pará con un grupo organizado de ciclistas aficionados que pedalean regularmente semanalmente y el estudio estuvo compuesto por 18 ciclistas. Como herramienta para la recopilación de datos, un cuestionario existente sobre calidad de vida utilizando el SF-36, que es un instrumento global creado por Ware y Sherbourne, y el cuestionario Nordic Muscle / Skeletal de Kourinka et al. Los datos obtenidos se analizaron mediante estadística descriptiva cuantitativa, mediante tablas de frecuencia. En la muestra, las mujeres tuvieron mayor participación que los hombres, ya que el $56 \%$ eran mujeres. Se concluye que el perfil de los ciclistas integrantes de la muestra muestra que las regiones anatómicas de la columna lumbar y la región de la rodilla fueron las áreas más predominantes para las quejas de dolor en comparación con las otras regiones y que la región torácica resultó ser una deficiente. área relevante para las lesiones en estos ciclistas amateurs y que la muestra no presentó dificultad o limitación en la práctica de sus actividades rigurosas del día o deportes.

Palabras clave: Ciclismo; Atletas aficionados; Lesiones musculoesqueléticos.

\section{Introdução}

O ciclismo é uma atividade esportiva bastante popular para as pessoas, isto por que é uma prática esportiva que possibilita uma melhora expressiva no condicionamento físico e por ser uma atividade de baixo impacto. A bicicleta é usada como meio de transporte e lazer de fácil acesso e, também, uma alternativa de locomoção favorável, além de ter um custobenefício aceitável e por promover uma boa saúde para seus praticantes (Alencar et al., 2012; Asplund \& Ross, 2012; Candotti, 2012). O ciclismo é responsável por proporcionar uma qualidade de vida para os que o praticam, por se tratar de uma atividade física que gera benefícios à saúde, como benefícios físicos, psicológicos e sociais (Krabbe \& Vargas, 2014; Oliveira et al., 2015).

Assim como toda modalidade esportiva, há também a possibilidade de surgir fatores que levam a um risco para possíveis lesões. No ciclismo não é diferente, pois, nessa modalidade, é exigido um mecanismo de repetição que pode causar uma lesão traumática ou não traumática (Coelho, 2016). Como é ressaltado também por Bernardo et al. (2012) e Barrios et al. (2015), as lesões nos ciclistas aparentam estar associada à lesões por acidentes e lesões não traumáticas por uso demasiado das estruturas.

Os ciclistas, tantos os atletas como os amadores, apresentam uma prevalência de lesões em regiões em comuns como: quadril, ombro, punho, períneo, joelho e região lombar. E destas regiões, as estruturas como o joelho e lombar são as mais provenientes de ocasionar lesões (Walt et al., 2014; Rodrigues et al., 2017).

A distribuição de carga exorbitante sobre as estruturas afetadas só evidencia a importância de uma bicicleta adequada, sendo que é um instrumento principal da prática do ciclismo, assim como os equipamentos necessários e que os mesmos devem proporcionar para os ciclistas um conforto adequado (Martins et al., 2016; Alencar et al., 2011 \& Stoelben et al. 2016).

Contudo, está pesquisa tem por finalidade levantar um perfil dos principais efeitos da pedalada em um grupo de ciclistas amadores da cidade de Santarém/Pará.

\section{Metodologia}

Trata-se de um estudo exploratório, analítico e descritivo com uma abordagem qualitativa e quantitativa. A pesquisa ocorreu na cidade de Santarém-Pará, com um grupo organizado de ciclistas amadores que pedalam de forma regular, semanalmente, e com mais de um ano de atuação. A amostra foi composta por 18 ciclistas de ambos os gêneros com idade entre 18 a 59 anos e que fazem parte de forma efetiva e disciplinar do grupo escolhido. Todos concordaram em participar da pesquisa mediante a assinatura do Termo de Consentimento Livre e Esclarecido (TCLE). Como critérios de inclusão, levou-se em consideração adultos que praticam o ciclismo amador com idade entre 18 e 59 anos; ambos os gêneros; atletas amadores que participam de forma regular do grupo praticando a pedalada semanalmente. Critérios de Exclusão: Não participaram do estudo ciclistas que não consentiram por meio do Termo de Consentimento Livre e Esclarecido (TCLE) a participação na 
pesquisa; os que tiveram antes ou durante a pesquisa algum dano físico, biológico e mental que as impossibilitaram de participar em alguma etapa da pesquisa.

A pesquisa foi realizada através de um breve questionário sobre qualidade de vida pelo SF-36 que é um instrumento global originalmente na língua inglesa norte-americana, mas que no Brasil sua tradução e validação cultural foi efetivada por Ciconelli et al. (1999). Foi aplicado também o questionário Nórdico Músculo/esquelético de Kourinka et al (1997) em que a versão traduzida e validada para população portuguesa é feita por Mesquita et al. (2010). Essa ferramenta avalia de forma fácil e acessível as queixas musculoesqueléticas apresentadas pelos participantes. Os questionários foram aplicados e enviados de forma on-line. Tendo como apoio metodológico foi utilizado o trabalho de Estrela, C. (2018), Metodologia Científica: Ciência, estudo, Ensino e pesquisa. Editora Artes Médicas.

A pesquisa obedece aos critérios propostos pela Resolução no 466, de 12 de dezembro de 2012 do Conselho Nacional de Saúde do Ministério da Saúde do Brasil que regulariza as pesquisas que envolvem seres humanos, sendo respeitados os princípios de autonomia, beneficência, não maledicência, justiça e equidade. A mesma foi aprovada pelo CEP do Centro Universitário Maurício Nassau, pelo número do parecer 4.939.124.

\section{Resultados e discussão}

Para atender os objetivos desse estudo, a pesquisa em questão contou com uma amostra de 18 participantes, como é descrito na Tabela 1 que demonstra a caracterização sociodemográfica desses participantes.

Tabela 1 - Caracterização sociodemográfica dos participantes.

\begin{tabular}{|cccc}
\hline Característica da amostra & & F & $\%$ \\
\hline Gênero & Feminino & 10 & $56 \%$ \\
\hline Masculino & 8 & $44 \%$ \\
\hline & 18 a 20 & 0 & $0 \%$ \\
\hline 21 a 30 & 1 & $6 \%$ \\
\hline & 30 a 40 & 7 & $39 \%$ \\
\hline & 40 a 50 & 8 & $44 \%$ \\
\hline
\end{tabular}

Fonte: Dados da pesquisa (2021).

A amostra da Tabela 1 refere-se ao gênero e idade destes voluntários em que é distribuída em frequência e porcentagem. Relacionado ao gênero dos integrantes que foi dada em masculino e feminino, nota-se que, o gênero feminino teve maior participação ativa do que o gênero masculino, uma vez que, 10 eram mulheres (56\%) e que 8 eram homens (44\%), apontando que a amostra é predominantemente feminina. Com relação às idades, percebe-se que teve um percentual maior com cerca de $44 \%$ dos participantes que apresentavam faixa etária de 40 a 50 anos de idade. Porém, a amostra também apresentou uma predominância na faixa etária de 30 a 40 anos, representando 39\% dos indivíduos. 
A Tabela 2 mostra as respostas dos ciclistas relacionadas as dores que apresentaram em nas regiões anatômica nos últimos 12 meses. Demostrando em frequência e porcentagem.

Tabela 2 - Respostas sobre as dores e as atividades nos últimos 12 meses (N: 18).

\begin{tabular}{|c|c|c|c|c|c|c|c|}
\hline \multirow[t]{2}{*}{ REGIÕES } & \multicolumn{4}{|c|}{$\begin{array}{l}\text { DOR NOS ÚLTIMOS } \\
12 \text { MESES? \% }\end{array}$} & \multicolumn{3}{|c|}{$\begin{array}{l}\text { ÚLTIMOS } 12 \text { MESES EVITOU } \\
\text { ATIVIDADES NORMAIS? \% }\end{array}$} \\
\hline & \multicolumn{2}{|c|}{ Sim } & \multicolumn{2}{|c|}{ Não } & Sim & \multicolumn{2}{|r|}{ Não } \\
\hline \multirow[t]{3}{*}{ Pescoço } & \multicolumn{2}{|c|}{5} & \multicolumn{2}{|c|}{13} & 1 & \multicolumn{2}{|r|}{17} \\
\hline & \multicolumn{2}{|c|}{$28 \%$} & \multicolumn{2}{|c|}{$72 \%$} & $5,6 \%$ & \multicolumn{2}{|r|}{$94,4 \%$} \\
\hline & Direito & Esquerdo & Ambos & Não & Direito & Esquerdo & Não \\
\hline \multirow[t]{3}{*}{ Ombros } & 2 & 3 & 2 & 11 & 1 & 3 & 14 \\
\hline & $11,1 \%$ & $16,7 \%$ & $11,1 \%$ & $61,1 \%$ & $5,6 \%$ & $16,7 \%$ & $77,8 \%$ \\
\hline & Direito & & rdo & Não & Direito & Esquerdo & Não \\
\hline \multirow[t]{2}{*}{ Cotovelos } & 1 & \multicolumn{2}{|c|}{1} & 16 & 0 & 1 & 17 \\
\hline & $5,6 \%$ & \multicolumn{2}{|c|}{$5,6 \%$} & $88,9 \%$ & $0,0 \%$ & $5,6 \%$ & $94,4 \%$ \\
\hline \multirow{3}{*}{ Punhos e Mãos } & Direito & Esquerdo & Ambos & Não & Direito & Esquerdo & Não \\
\hline & 3 & 2 & 2 & 11 & 1 & 1 & 16 \\
\hline & $16,7 \%$ & $11,1 \%$ & $11,1 \%$ & $61,1 \%$ & $5,6 \%$ & $5,6 \%$ & $88,9 \%$ \\
\hline \multirow{3}{*}{ Torácica } & \multicolumn{2}{|c|}{ Sim } & \multicolumn{2}{|c|}{ Não } & \multicolumn{2}{|l|}{ Sim } & Não \\
\hline & \multicolumn{2}{|c|}{0} & \multicolumn{2}{|c|}{18} & \multicolumn{2}{|l|}{0} & 18 \\
\hline & \multicolumn{2}{|c|}{$0 \%$} & \multicolumn{2}{|c|}{$100 \%$} & $0 \%$ & & $100 \%$ \\
\hline & & & & & Sim & & Não \\
\hline Lombar & & & & & 5 & & 13 \\
\hline & & & & & $28 \%$ & & $72 \%$ \\
\hline & Esquerdo & & & Não & Esquerdo & & Não \\
\hline एवatain & 1 & & & 15 & 3 & & 15 \\
\hline & $5,6 \%$ & & & $83,3 \%$ & $27,8 \%$ & & $72,2 \%$ \\
\hline & Esquerdo & & & Não & Esquerdo & & Não \\
\hline Glúteos e coxa & 3 & & & 14 & 3 & & 15 \\
\hline & $16,7 \%$ & & & $77,8 \%$ & $16,7 \%$ & & $83,3 \%$ \\
\hline & Direito & Esquerdo & Ambos & Não & Direito & & Não \\
\hline Joenos & 5 & 4 & 1 & 8 & 3 & & 15 \\
\hline & $27,8 \%$ & $22,2 \%$ & $5,6 \%$ & $44,4 \%$ & $16,7 \%$ & & $83,3 \%$ \\
\hline & Direito & & erdo & Não & Direito & & Não \\
\hline Tornozelo e Pé & & & & & & & \\
\hline & 1 & & & 16 & 2 & & 16 \\
\hline & $5,6 \%$ & & & $88,9 \%$ & $11 \%$ & & $89 \%$ \\
\hline
\end{tabular}

Fonte: Dados da pesquisa (2021). 
Analisando a Tabela 2, observa-se que os participantes relataram que as regiões que mais apresentam sintomas de dor, foram as regiões de joelho e lombar. Da amostra 10 ciclistas relataram que sentiram dor nos últimos 12 meses representando $(55,6 \%)$ destes ciclistas, sendo que $5(27,8 \%)$ no joelho direito, $4(22,2 \%)$ em joelho esquerdo e $1(5,6 \%)$ respondeu em ambos os joelhos. Em seu estudo Fortuna et al. (2017) destacou-se que a região dos joelhos também apresentou uma prevalência de dor superior ao se comparar com demais regiões com $44 \%$ em sua porcentagem. Entretanto, é interessante ressaltar que ao comparar com o estudo atual, o percentual dos ciclistas que reportaram a dor só na região do joelho, difere do estudo de Fortuna et al. (2017), já que a amostra do estudo atual, conforme a tabela 2, passa a ser maior, e no estudo de Walt et al. (2014) $42 \%$ reportaram dor em joelhos e riscos de lesões.

Com relação a região lombar, 10 dos participantes (56\%), ou seja, metade da amostra, declararam que sofrem de dores nesta região e apenas 8 dos participantes (44\%) relataram que não sentiram. Estes resultados vão de acordo com o estudo de Rodrigues et al. (2017), já que 30\% a 60\% dos ciclistas sentem dor na área lombar, resultando em um quadro de lombalgia, que segundo Kotler et al. (2016), a lombalgia pode estar relacionada com doenças degenerativas do disco vertebral ou devido a um fator importante que é flexão excessiva exercida na bicicleta durante a pedalada. Vale destacar que 3 (16,7\%) dos ciclistas tiveram que evitar suas atividades normais e $15(83,3 \%)$ não tiveram para a região do joelho; para a lombar 5 (28\%) responderam que sim e $13(72 \%)$ que não, é o que se confirma com o estudo de Fortuna et al. (2017) onde 20\% responderam sim para a estrutura do joelho e 13,3\% para lombar.

Ao examinar a tabela 2, as regiões do ombro, punhos e mãos, que comparada com outras regiões também apresentaram respostas consideráveis, no qual 7 reportaram dor no ombro, onde $2(11,1 \%)$ reportaram em ombro direito, 3 (16\%) em esquerdo e $2(11,1 \%)$ em ambos. Nos punhos e mãos, 7 (38,9\%) dos 18 ciclistas representando (61\%) disseram sentir dor; $3(16 \%)$ direito, $2(11,1 \%)$ esquerdo e $2(11,1 \%)$ em ambos.

A respeito da região torácica, o estudo atual mostrou que os indivíduos não tiveram sintomatologia dolorosa nessa estrutura e que mostra ser uma área de menos relevância para lesão nos ciclistas, Porém, Dahlquist et al. (2015) em sua pesquisa demonstrou que pelo menos $30,2 \%$ dos ciclistas apontaram sentir dores nesta região.

O Gráfico1 abaixo refere-se as atividades que exigem esforço físico, tais como correr, levantar objetos pesados e participar de esportes árduos.

Gráfico 1 - Resposta dos ciclistas relacionadas a atividades rigorosas.

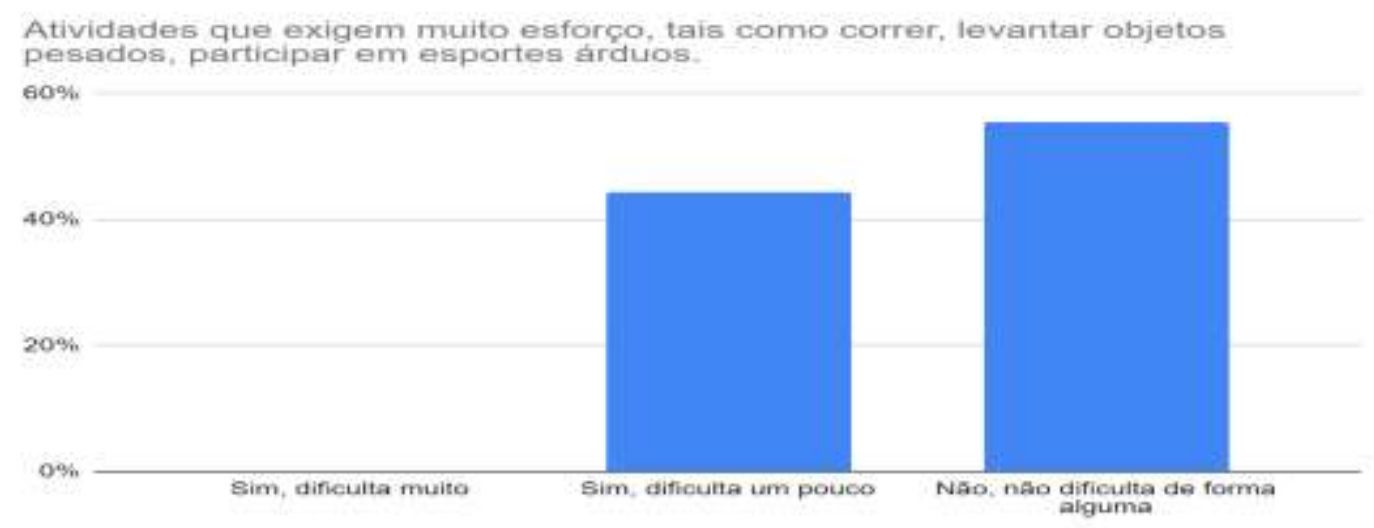

Fonte: Dados da pesquisa (2021).

Nota-se que a maioria respondeu não sentir dificuldades ao realizar suas atividades, nesse caso, 10 participantes que representam 56\% da amostra, mas que 8 dos participantes, o que representa 44\%, apresentaram sentir leve dificuldade em realizar suas atividades. Vale ressaltar que a maior parte da amostra demonstrou não ter nenhuma dificuldade ou limitação em 
praticar suas atividades rigorosas do dia a dia ou modalidades esportivas. De acordo com estudo Nunes et al. (2017), 33 dos participantes de sua pesquisa também não relataram nenhuma dificuldade em realizar suas atividades.

Com relação ao Gráfico 2 refere-se aos ciclistas que relataram problemas em alguma região do corpo nos últimos 7 dias.

Gráfico 2 - Respostas dos ciclistas que relataram dor nos últimos 7 dias.

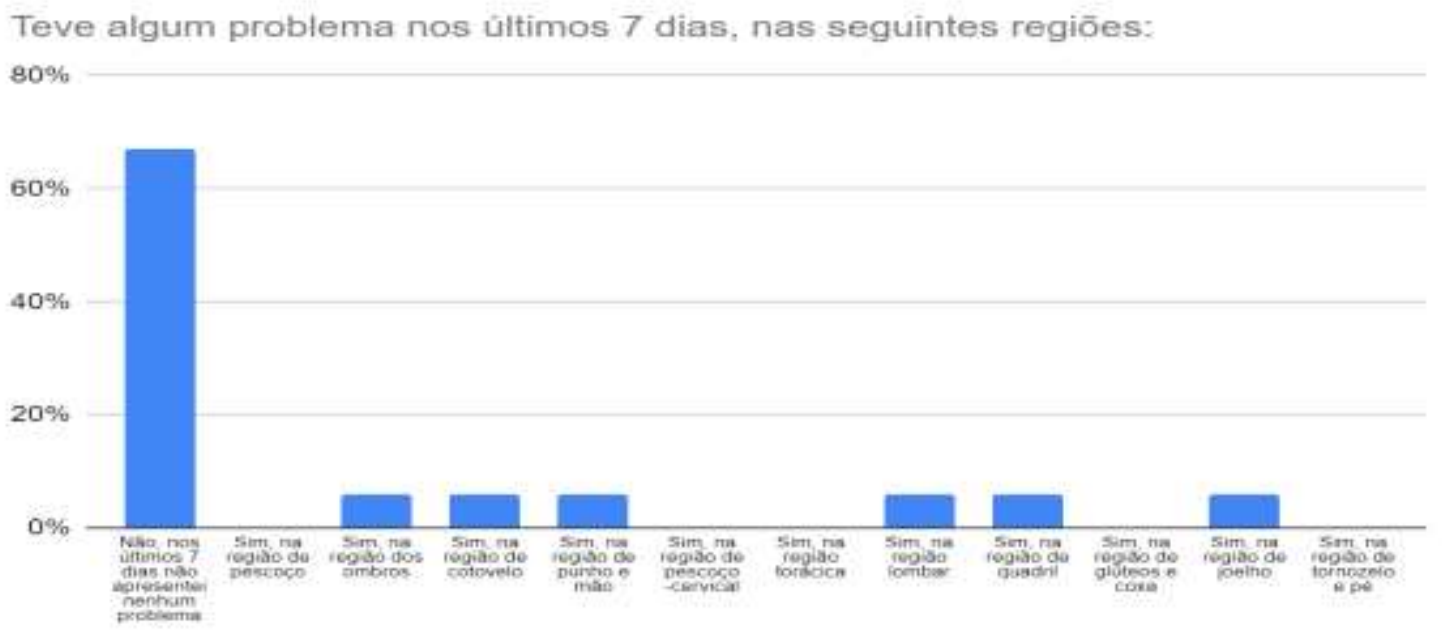

Fonte: Dados da pesquisa (2021)

Observou-se que, a grande maioria, no total de 12 pessoas (67\%), não apresentou nenhum problema. Porém, 6\% dos participantes relataram sentir problemas na região dos ombros; $6 \%$ na região do cotovelo; $6 \%$ na região de punho e mão; $6 \%$ na região lombar; $6 \%$ na região de quadril e outros $6 \%$ relataram sentir na região de joelho. Em seu estudo, Fortuna et al. (2017) relata que a grande maioria $(43,30 \%)$ sentiu dor na região lombar e nos joelhos. Quanto à região de ombro, foi possível observar que ambos (10\% direito e 10\% esquerdo) sentiram dor nos últimos 7 dias, evitando suas atividades de vida diária. Quanto às regiões de punho/mão e região torácica, Fortuna et al. (2017) observou que também foram regiões acometidas pela dor e com isso optaram por também assim evitar suas atividades de vida diária.

\section{Conclusão}

Conclui-se com esse estudo que, o perfil dos ciclistas que compõem à amostra apresentou as seguintes características, mostrando que, as regiões anatômicas da coluna lombar e as regiões dos joelhos foram as áreas que mais predominaram para queixa de dores ao se comparar com as demais regiões.

Outro achado importante desse estudo, foi que na região torácica, os indivíduos não manifestaram nenhuma queixa dolorosa dessa estrutura, o que mostrou ser uma área pouco relevante para lesões nesses ciclistas amadores. Com relação às atividades rigorosas que os integrantes da amostra exercem, notou-se que a maioria não apresenta limitações ou dificuldades para realizá-las. Isso demonstra que os ciclistas amadores apresentam uma resposta positiva para sua qualidade de vida. É necessário ressaltar a importância do desenvolvimento de trabalhos futuros relacionados a este tema, principalmente sobre as possíveis lesões patológicas que venham a surgir nas regiões anatômicas que obtiveram maiores queixas dolorosas pelos ciclistas amadores. 


\section{Agradecimentos}

Primeiramente agradecemos a Deus por nos ter dado a determinação e o entendimento necessário para concluir esse trabalho e ao nosso orientador Assis Pantoja por ter nos ajudado durante essa caminhada.

\section{Referências}

Alencar, T. A. M. D., Matias, K. F. S. D., \& Aguiar, B. C. D. (2012). Lesões agudas em ciclistas. Revista movimenta issn: 1984-4298 v. 5, n. 3.

Alencar, E. T. A. et al. (2011). Revisão etiológica da lombalgia em ciclista. Revista Brasileira ciência do esporte, Florianópolis.

Asplund, C.; \& Ross, M. (2012). Core Stability And Bicycling. Revista Current sports medicine reports, v. 9, n. 3, p. $155-160$.

Barrios, C., Bernardo, N. D., Vera, P., Laíz, C., \& Hadala, M. (2015). Changes in sports injuries incidence over time in world-class road cyclists. Revista International journal of sports medicine, 36(03), 241-248.

Bernardo, N., Barrios, C., Vera, P., Laíz, C., \& Hadala, M. (2012). Incidence and risk for traumatic and overuse injuries in top-level road cyclists. Journal of sports sciences, 30(10), 1047-1053.

Candotti, C. T., Schaurich, R. F., Torre, M., Noll, M., Pasini M., \& Loss J. F. (2012). Atividade elétrica e força muscular dos extensores cervicais durante o ciclismo. 1: 40- 50

Ciconelli, R.M., Ferraz, M. B., Santos, W., \& Meinão, I. (1999). Quaresma, M.R. Tradução para a língua portuguesa e validação do questionário genérico de avaliação da qualidade de vida SF-36 (Brasil SF-36). Revista Brasileira de Reumatologia, São Paulo, v.39, n.3, p.143-50.

Coelho, T. B. (2016). O efeito do bikefit no desempenho de ciclistas e na prevenção de lesões não traumáticas associadas ao ciclismo. Dissertação (medicina) Universidade da beira interior, Covilhã- Portugal.

Dahlquist, M., Leisz, M. C., \& Finkelstein, M. (2015). The club-level road cyclist: injury, pain, and performance. Clinical journal of sport medicine, 25(2), 8894.

Fortuna, P., Cervaens, M., \& Pereira, P. (2017). Prevalência de lesões musculo-esquelética em ciclistas. https://bdigital.ufp.pt/bitstream/10284/6287/1/PG_27800.

Kotler, D. H., Babu, A. N., \& Rrobidoux, G. (2016). Prevention, evaluation, and rehabilitation of cycling-related injury. Current sports medicine reports, 15(3), 199-206

Krabbe, S., \& Vargas, A. C. (2014). Qualidade de vida percebida por mulheres em diferentes tipos de exercício físico. Revista Kairós : Gerontologia, p. 193204.

Kuorinka, I., Jonsson, B., Kilbom, A., Vinterberg, H., Biering-Sørensen, F., Andersson, G., \& Jørgensen, K. (1987). Standardised Nordic questionnaires for the analysis of musculoskeletal symptoms. Applied ergonomics, 18(3), 233-237.

Martins, A. S., Santos, M. G. R., Junior, J. R. S., \& Lemos, T. V. (2016). Avaliação Fisioterapêutica no ciclismo: um estudo de caso. Revista eletrônica de trabalhos acadêmicos, área da saúde.

Mesquita, C. C., Ribeiro, J. C., \& Moreira, P. (2010). Portuguese version of the standardized Nordic musculoskeletal questionnaire: cross cultural and reliability. Journal of Public Health, 18(5), 461-466.

Nunes, K., \& Fonseca, P.D. S. (2017). Prevalência de lesões osteomioarticulares em ciclistas amadores. http://rdu.unicesumar.edu.br/handle/123456789/394.

Oliveira, J. D. F. et al. (2015). Avaliação da qualidade de vida de um grupo de tabagistas no processo de cessação do tabaco: a questão da atividade física. Salusvita. Bauru.

Rodrigues, A.C., Costa, C. A., Porto, V. H. B., \& Ribeiro, D. S. (2017). Prevalência de dor lombar em ciclistas praticantes em goiás, amadores das modalidades: mountain bike, time trial e road. Revista científica multidisciplinar núcleo do conhecimento v.15, n.2, p.235-246.

Stoelben, k. J. V.; Silva, f. S.; Lemos, j. C., \& Mota, c. B. (2016). Comportamento do Ângulo q e ângulo de adução do quadril na pedalada de ciclistas: um estudo piloto. Revista educação física, j phys ed. 85, 3, 257-26.

Walt, A., Janse van Rensburg, D. C., Fletcher, L., Grant, C. C., \& Walt, A. J. (2014). Non-traumatic injury profile of amateur cyclists. South African Journal of Sports Medicine, 26(4), 119-122. 